\title{
Description of fission yields in the nucleon-induced fission reactions
}

\author{
S. Yavshits ${ }^{1, a}$ and O. Grudzevich ${ }^{2}$ \\ ${ }^{1}$ Khlopin Radium Institute, 194021 St.-Petersburg, Russia \\ 2 Obninsk State Technical University, Obninsk, Russia
}

\begin{abstract}
The potential model for the fission fragment mass distributions and simplified approach for the isobaric charge distribution are proposed for the description of fission yields (FY). The intermediate energy reaction code MCFx was used for the calculation of the fissionning nuclei distribution after fast (cascade), preequilibrium and statistical reaction stages. Formation of the mass distributions is considered as a result of oscillations on mass asymmetry degree of freedom in the potential well calculated with the temperature dependent shell correction method. The comparison of calculation results with the experimental data on FY for both low and intermediate energy fission show a good agreement of the data and let us conclude that the approach proposed may be useful for FY evaluations for experimentally unknown fission yields data in the case of the intermediate energy nucleon-induced fission.
\end{abstract}

\section{Introduction}

Formation of fragments in the nuclear fission is closely tied with the important and still incompletely studied process of the nuclear matter fragmentation from both low excited (spontaneous and thermal fission) and excited and highly excited states in the cases of intermediate and high energy nucleon-induced fission. Instability of outward fission barrier on octupole shape deformations has been noted already in the classical work [1]. However, until recently mass spectra of fission fragments were studied rather qualitatively. A number of sophisticated microscopic analyses [2-6] allowed to establish main characteristics of the process but these models are unpractical for systematical description of fragment mass spectra and nuclear data generation. Generalization of multimodal random neck rupture model to take into account nuclear temperature effects on fragment fission formation was presented in the work [7]. Results of calculation performed in this work shown a rather well description of experimental data for both low and intermediate energy fission. The analysis of contributions of different fission modes into the fragment mass spectra in the framework of multimodal model has been also done in the work [8].

\section{Model}

One of the important points in the study of nuclear configurations near the scission point is the choice of shape parameterization. We used the method proposed by V. Pashkevich [9] for axially symmetrical configurations. Here nuclear shape is defined in the orthogonal coordinate system where base family of coordinate surfaces is deformed Cassini ovaloids allowing to describe both oblate and prolate shapes including strongly prolate ones right up to division of nucleus on two fragments. This shape parameterization is especially suitable for fission and heavy ion reactions research but could be also applied for shapes near sphere. Let us restrict the consideration

\footnotetext{
${ }^{a}$ Presenting author, e-mail: email@address.zz
}

by three main parameters of deformations that are $\{\alpha\}=$ $\left(\alpha, \alpha_{1}, \alpha_{4}\right)$ where $\alpha$ is the lemniscate parameter, $\alpha_{1}$ defines mirror symmetry of nuclear shape and $\alpha_{4}$ is the parameter of hexadecapole deformations. At small values of the lemniscate parameter the shape of nucleus looks like ovaloids while values $\alpha>0.9$ correspond to configurations with developed neck.

Collective motion in the space of collective coordinates is defined in the common case by dynamical and static nuclear properties. Fission process as a process of movement from ground state to scission point is the large-scale nuclear process, the properties of fission depending both from the links of single particle degrees of freedom with collective coordinates which are manifested as friction, from fluctuations of an effective mass coupled with coordinate $\alpha$ and other effects and the structure of potential energy surface. One can expect that for formation of fragment mass asymmetry is defined mainly by properties of deformation energy near the scission because observed displacements from symmetrical fission are not very strong (about $10-15 \%$ percents for actinides).

We use one-dimensional Schrödinger equation for description of the collective motion over mass-asymmetry coordinate near the scission point:

$$
\left\{-\frac{\hbar^{2}}{2 \sqrt{\mathrm{B}}_{1}} \frac{\partial}{\partial \alpha_{1}} \frac{1}{\sqrt{\mathrm{B}}_{1}} \frac{\partial}{\partial \alpha_{1}}+\mathrm{V}_{\mathrm{T}}\left(\alpha_{1} ; \alpha_{\mathrm{sc}} ; \alpha_{4}^{\min }\right)\right\} \psi_{v}\left(\alpha_{1}\right)=\mathrm{E}_{\nu} \psi\left(\alpha_{1}\right) .
$$

Here $B_{1}$ is the mass parameter for mass-asymmetry mode $\alpha_{1}, V_{T}\left(\alpha_{1} ; \alpha_{s c} ; \alpha_{4}^{\mathrm{min}}\right)$ is the temperature dependent collective potential energy of deformation as function of $\alpha_{1}$ at the scission point $\alpha=\alpha_{s c}, \alpha_{4}^{\min }$ is chosen from the condition of potential energy minima, $\psi_{v}\left(\alpha_{1}\right)$ are collective wave functions and $E_{v}$ is the energy spectra of collective states.

Due to Strutinsky's prescription [10] the potential energy in the space of collective parameters $\{\alpha\}$ could be presented as a sum of smooth liquid drop part and shell and pair corrections:

$$
V_{T}(\{\alpha\})=E_{l d}(\{\alpha\})+f(T) \delta E(\{\alpha\}),
$$


where $E_{l d}$ is a liquid drop energy, quantity $\delta E$ takes into account shell correction and pair energy, $\delta E=E_{\text {shell }}+E_{\text {pair }}$ and function $f(T)$ reflects dumping of nuclear structure effects with nuclear temperature. We use Woods-Saxon form of dumping function $f(T)=1 /\left(1+\exp \left(T-T_{c r}\right) / \alpha\right)$, where $T_{c r}$ and $\alpha$ are the model parameters. Single particle spectra which are necessary to calculate shell corrections and pair energies have been calculated with DIANA code [11], the nuclear shape has being taken in the lemniscate coordinate and mean field potential was as deformed Woods-Saxon potential.

Probability to find configuration with given value of $\alpha_{1}$ can be expressed as follows:

$$
Y\left(\alpha_{1}\right) \propto \sum_{v}\left|\Psi_{v}\left(\alpha_{1}\right)\right|^{2} e^{-E v / T} d \alpha_{1},
$$

while fragment mass spectra has the following form:

$$
Y\left(A_{1}\left(\alpha_{1}\right)\right) \propto Y\left(\alpha_{1}\right) \frac{d A_{1}}{d \alpha_{1}},
$$

where $A_{1}$ is the mass number of one from the fragments.

Let us define main quantities in the equation (1). One of the important questions here is the definition of scission point. We suggest that scission takes place when neck radius is equal to $1 \mathrm{fm}$ that is about nucleon radius. Such a condition allows to fix lemniscate parameter $\alpha_{s c} \approx 0.99$ (value of $\alpha_{s c}$ is slightly varied in dependence of mass number of fissioning nucleus).

Dynamical nuclear properties are reflected in the behavior of mass parameter $B_{1}$ as function of $\alpha_{1}$. General microscopical calculation of effective mass as a response of nuclear system on the mean field distortions is a real challenge enclosing a number of ambiguities. In our simplified approach we used liquid drop value for small oscillations $B_{1} \propto A^{5 / 3}$.

For intermediate-energy fission one should take into account the formation of wide distribution of fissioning nuclei on mass and charge numbers and excitation energies $A_{f}$, $Z_{f}, E_{f}^{*}$ due to prefission particle emission at each stage of nucleon-induced reaction, i.e., at direct, preequilibrium and equilibrium (multichance fission) stages. The final fragment mass distribution could be found as a superposition of fragment yields $Y_{f}$ for each nucleus with corresponding weight $W\left(A_{f}, Z_{f}, E^{*}\right)$ defined by the reaction mechanism:

$$
Y \propto \sum_{A_{f}, Z_{f}, E^{*}} W\left(A_{f}, Z_{f}, E^{*}\right) Y_{f} .
$$

The calculations of weights have been done with the code MCFx developed earlier by us [12].

\section{Results}

The profile of potential energy as function of fission fragment mass asymmetry is shown in the figure 1. Link between asymmetry parameter $\alpha_{1}$ and fragment mass was found as the following integral:

$$
A_{1}=2 \pi \frac{A_{f}}{V} \int_{z_{\min }}^{z 1} r^{2}(z) d z
$$

where $A_{1}$ is the mass number of one of fragments, $V$ is the volume of fissioning nucleus, correspondingly, $r(z)$ is the

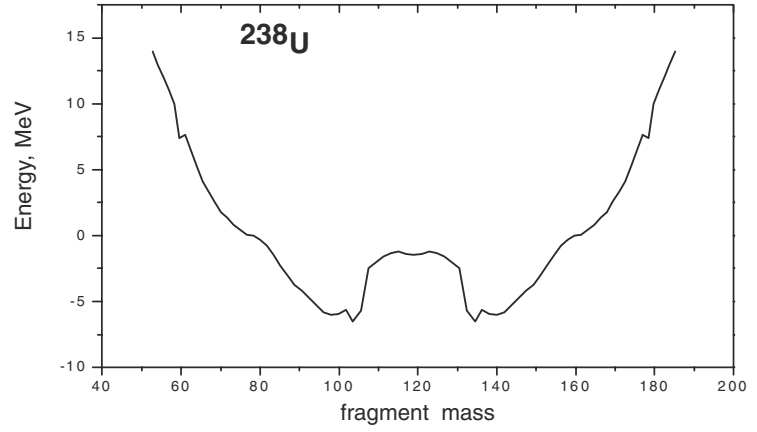

Fig. 1. Potential energy of deformation in the scission point for the case of ${ }^{238} \mathrm{U}$ fission as function of nascent fragment mass number.

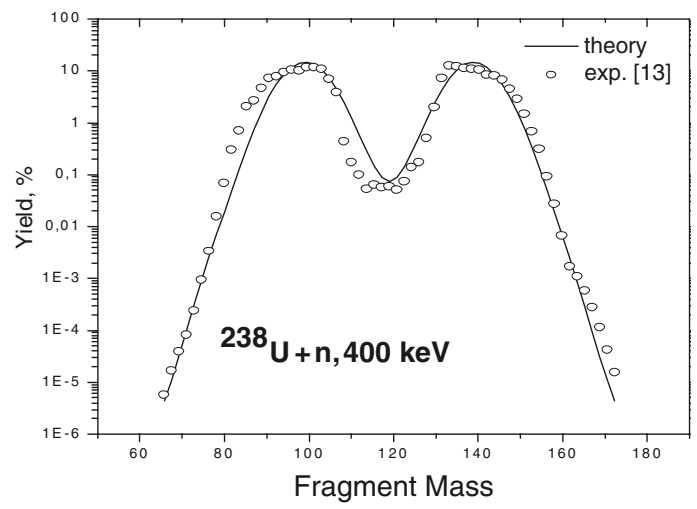

Fig. 2. Fission fragment yields for low energy neutron-induced fission of ${ }^{238} \mathrm{U}$.

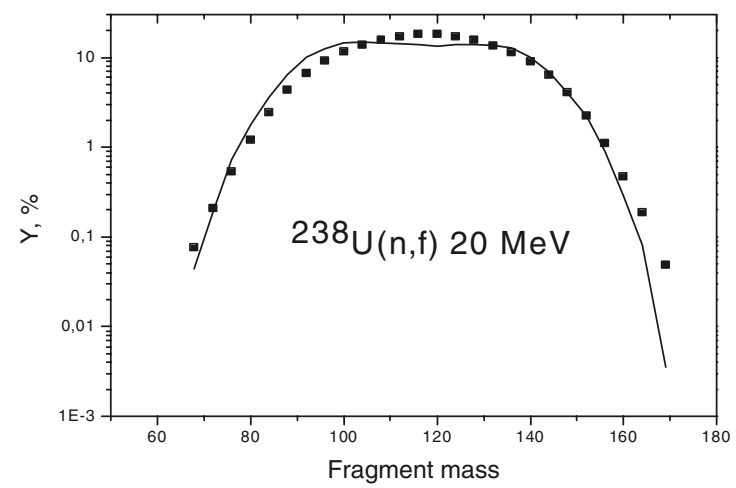

Fig. 3. Fission fragment yields for $20 \mathrm{MeV}$ neutron-induced fission of ${ }^{238} \mathrm{U}$. The experimental data (squares) were taken from ref. [14].

surface equation in cylindrical coordinates $(r, z), z_{\min }$ is the value of $z$ coordinate in the point with the minimal value of neck radius, and $z_{1}$ is the value of $z$ at edge of nucleus.

It is seen from the figure 1 that results of our calculations shows clear minimum in the potential energy near fragment mass 140 a.u. in accordance with well-known property of fragment mass distributions of actinides.

The solution of equation (1) has been found as series expansion on the oscillator basis. Our results for lowenergy fission fragment mass distributions are presented in the figure 2.

Results for the intermediate energy fission are presented in figures 3-6 for neutron- and proton-induced fission in comparison with the experimental data. 


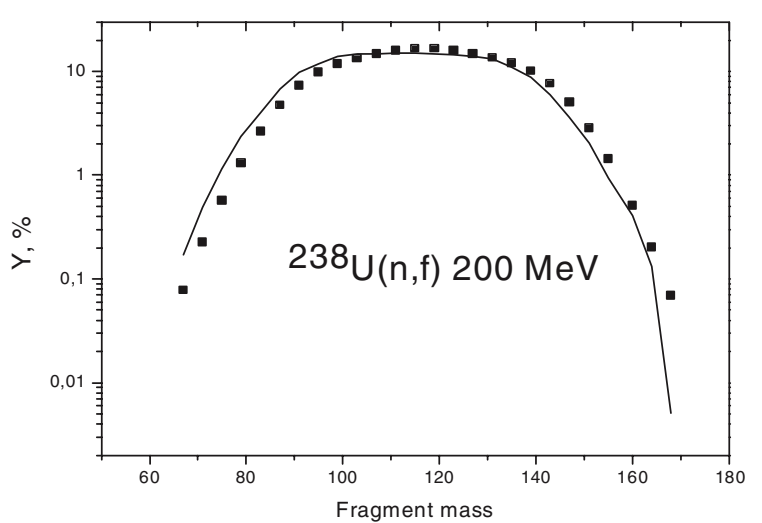

Fig. 4. Fission fragment yields for $200 \mathrm{MeV}$ neutron-induced fission of ${ }^{238} \mathrm{U}$. The experimental data (squares) were taken from ref. [14].

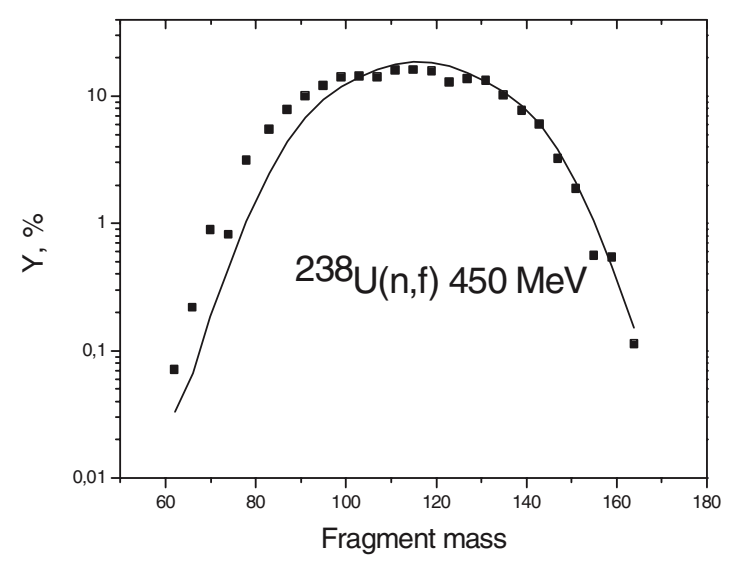

Fig. 5. Fission fragment yields for $450 \mathrm{MeV}$ neutron-induced fission of ${ }^{238} \mathrm{U}$. The experimental data (squares) were taken from ref. [14].

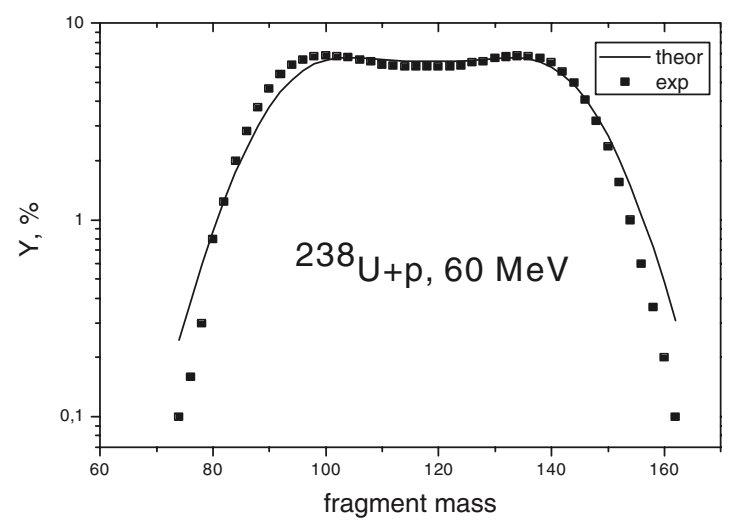

Fig. 6. Fission fragment yields for $60 \mathrm{MeV}$ proton-induced fission of ${ }^{238} \mathrm{U}$. The experimental data (squares) were taken from ref. [8].

One can see that in these cases our results reproduce experimental data rather well.

Some results of isobaric charge distributions are presented in figures 7-8. The simple approximation was used in this case

$$
Y_{Z}(A) \propto \exp \left[\frac{\left(A-\bar{A}_{z}^{2}\right.}{2 \sigma_{A}^{2}}\right],
$$

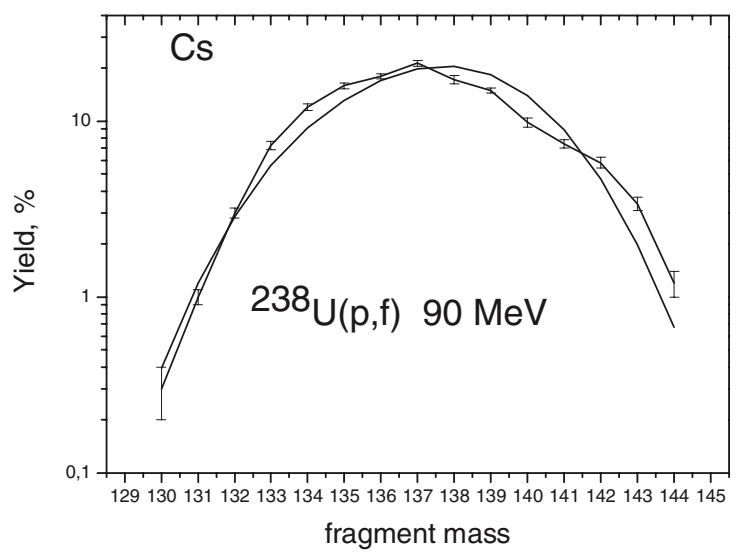

Fig. 7. Yields of Cs isotopes for $90 \mathrm{MeV}$ proton-induced fission of ${ }^{238} \mathrm{U}$. The experimental data were taken from ref. [16].

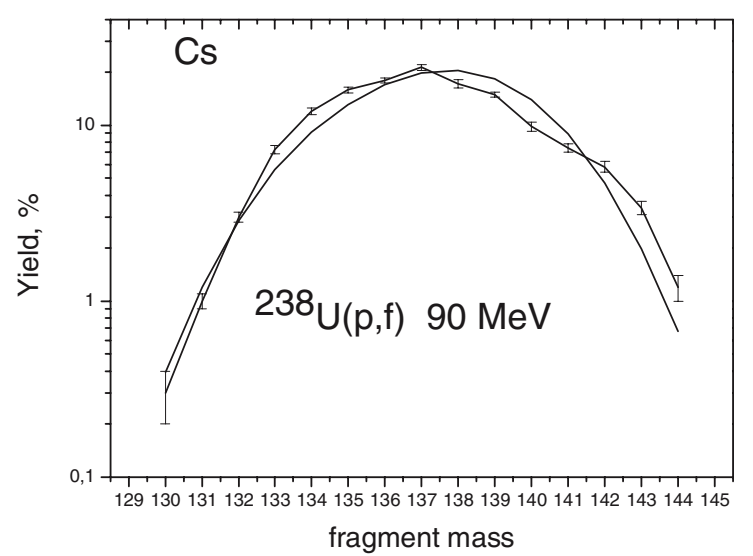

Fig. 8. Yields of $\mathrm{Rb}$ isotopes for $90 \mathrm{MeV}$ proton-induced fission of ${ }^{238} \mathrm{U}$. The experimental data were taken from ref. [16].

where $\bar{A}=(Z+\delta Z) \frac{A_{f}}{Z_{f}}-v, \sigma_{A}^{2}=\sigma_{z}^{2}\left(\frac{A_{f}}{Z_{f}}\right)^{2}, A$ and $Z$ are mass and charge number of the fragment, $v$ is the number of neutrons emitted from the fragment accordingly [8] and values of $\sigma_{z}^{2}=0.4, \delta Z=0.5$ were taken from the experimental data for low-energy fission. It is seen from the figures that the calculations results are in the good agreement with the experimental data [16].

So, we can conclude that the model developed in link with MCFx code can be used for data file generation on the fission fragment mass distributions at low- and intermediate energy fission.

\section{References}

1. M. Brack, J. Damgaard, A. Jensen et al., Rev. Mod. Phys. 44, 320 (1972).

2. J.W. Negele, S.E. Koonin, P. Moller et al., Phys. Rev. C 17, 1098 (1978).

3. J.-F. Berger, M. Girod, D. Gogny, Nucl. Phys. A 428, 23c (1984).

4. G.D. Adeev, I.I. Gonchar, L.A. Marchenko et al., Sov. J. Nucl. Phys. 43, 727 (1986). 
5. S.K. Samaddar, D. Sperber, M. Zielinska-Pfabe et al., Phys. Scr. 25, 517 (1982)

6. H. Goutte, J.-F. Berger, P. Casoli, D. Gogny, Phys. Rev. C 71 , 024316 (2005).

7. M.C. Duijvestijn, A.J. Koning, F.-J. Hambsch, Phys. Rev. C 64, 014607 (2001).

8. V.A. Rubchenya, J. Aysto, Nucl. Phys. A 701, 127c (2002).

9. V.V. Pashkevich, Nucl. Phys. A 169, 275 (1969).

10. V.M. Strutinsky, Nucl. Phys. A 122, 1 (1968).
11. V.A. Rubchenya, V.V. Pashkevich, Bull. of Nuclear Data Center LIYAF 3, 3 (1976).

12. S.Yavshits, Report IAEA-NDS-153, 2002.

13. JEFF 2.2 Report AEA-1015, 1993.

14. C.M. Zoller, Ph.D. thesis, TH Darmstadt, 1995.

15. F. Göennenwein, in The Nuclear Fission Process, edited by C. Wagemans (CRC Press, London, 1991).

16. B.N. Belyaev, V.D. Domkin, V.S. Mukhin, Yadernaya Fizika 57, 1231 (1994). 